\title{
NÍVEIS DE ALUMÍNIO E ACÚMULO DE MACRONUTRIENTES EM PORTA-ENXERTOS CÍTRICOS EM CULTIVO HIDROPÔNICO ${ }^{1}$
} \author{
Zambello de Pinho ${ }^{4}$ \\ ${ }^{2}$ Depto. de Botânica - IB/UNESP, C.P. 502 - CEP: 18618-000 - Botucatu, SP. \\ ${ }^{3}$ Depto. de Ciência do Solo - FCA/UNESP, C.P. 237 - CEP: 18603-970 - Botucatu, SP. \\ ${ }^{4}$ Depto. de Bioestatística - IB/UNESP. \\ *e-mail: heliograssi@fca.unesp.br
}

Carlos Henrique dos Santos²; Hélio Grassi Filho ${ }^{3 *}$; João Domingos Rodrigues²; Sheila

RESUMO: O presente trabalho teve como objetivo avaliar a influência de diferentes níveis de alumínio no acúmulo de macronutrientes pelos porta-enxertos cítricos limoeiro Cravo e citrumeleiro Swingle, sob cultivo hidropônico. Os tratamentos utilizados em condições de casa de vegetação seguiram o delineamento estatístico inteiramente casualizado, com 3 repetições, em parcelas subdivididas. Os níveis de alumínio utilizados foram: $0,7,5,15,22,5$ e $30 \mathrm{mg} \mathrm{L}^{-1}$, na forma de $\mathrm{AlCl}_{3} .6 \mathrm{H}_{2} \mathrm{O}$. Determinaramse o acúmulo de $\mathrm{N}, \mathrm{P}, \mathrm{K}, \mathrm{Ca}, \mathrm{Mg}, \mathrm{S}$ e do $\mathrm{Al}$ da matéria seca vegetal, relacionando-os com os pesos da matéria seca da parte aérea (folhas e caule) e raízes durante o período de 84 dias, com coletas de plantas a cada 14 dias. Conclui-se que, com adição a partir de $7,5 \mathrm{mg} \mathrm{L}^{-1}$ de alumínio em solução nutritiva ocorreram alterações fisiológicas e reduções significativas no acúmulo de macronutrientes associado à redução nos pesos da matéria seca das folhas, caule e raízes das plantas de citrumelo Swingle.

Palavras-chave: Citrus, porta-enxerto, alumínio, nutrição mineral, macronutriente

\section{ALUMINUM LEVELS AND MACRONUTRIENT ACCUMULATION IN CITRUS ROOTSTOCKS IN HYDROPONIC SOLUTION}

ABSTRACT: The aim of this research was to study the influence of different levels of aluminum on macronutrient contents of citrus rootstocks of the 'Rangpur' lime and the 'Swingle' citrumelo, in hydroponic solution. The treatments, established under greenhouse conditions, followed a completely randomized experimental design, with 3 replications, distributed in split-plots. The levels of aluminum were: $0 ; 7.5 ; 15$; 22.5 and $30 \mathrm{mg} \mathrm{L}^{-1}$, applied as $\mathrm{AlCl}_{3} \cdot 6 \mathrm{H}_{2} \mathrm{O}$. The evaluated chemical plant parameters were the concentrations of $\mathrm{N}, \mathrm{P}, \mathrm{K}, \mathrm{Ca}, \mathrm{Mg}, \mathrm{S}, \mathrm{Al}$ and dry weight of leaves, stem and root system. The results showed that with adition of $7.5 \mathrm{mg} \mathrm{L}^{-1}$ of $A$ l to the nutrient solution there was a significant decrese of nutrient contents and a decrese in leave, stem and root system dry weight of 'Swingle' citrumelo.

Key words: Citrus, rootstocks, aluminum, mineral nutrition, macronutrient

\section{INTRODUÇÃO}

Apesar da expansão da citricultura e do aumento da produção ocorridos ao longo dos anos, segundo Amaro \& Maia (1997), não houve incremento na produtividade a ponto de justificar o crescimento da produção, sendo que a escolha do porta-enxerto e a nutrição das plantas cítricas podem contribuir para esta realidade.

O Brasil possui condições favoráveis para a expansão da cultura dos citros, entretanto tem sido exploradas áreas de solos ácidos, de baixa fertilidade e com elevados teores de alumínio
(Vasconcellos, 1987 e Nogueira et al., 1989).

O alumínio, tem efeito tóxico quando em quantidade elevada na solução do solo, limitando o crescimento de muitas espécies de plantas embora algumas sejam beneficiadas com a sua presença (Foy et al., 1978).

Alguns trabalhos de pesquisa sobre a toxidez de alumínio nas plantas mostraram que este fixa o fósforo em formas menos solúveis no solo ou nas raízes, e ainda pode interferir na absorção, transporte e utilização de vários nutrientes como $\mathrm{Ca}, \mathrm{Mg}, \mathrm{P}, \mathrm{K}$ e na água usada pelas plantas (Foy, 1976).

${ }^{1}$ Parte da Dissertação de Mestrado do primeiro autor apresentada à FCA/UNESP - Botucatu, SP. 
Variedades promissoras sob determinadas condições edafoclimáticas têm sido indicadas pela pesquisa, visando promover a diversificação de porta-enxertos nos pomares comerciais. Entretanto, esta diversificação não tem ocorrido com a amplitude necessária, sendo - limoeiro Cravo ainda o mais utilizado (Pompeu Júnior, 1991). Outro fator a ser considerado, é que existem poucas informações sobre a assimilação de nutrientes por diferentes portaenxertos relacionada à tolerância de alumínio em regiões tropicais, principalmente no Brasil onde a citricultura é de grande importância econômica (Vasconcellos, 1987).

Assim sendo, o objetivo do presente trabalho é obter informações a respeito da influência do alumínio no acúmulo de macronutrientes das plantas de dois portaenxertos. A escolha do limoeiro Cravo deve-se às suas excepcionais características, destacando-o como padrão nacional. Por outro lado, confirmando-se a atual tendência de cotação dos preços da laranja baseada no maior teor de sólidos solúveis do fruto, inclui-se o citrumeleiro Swingle, visto ser esta uma característica superior do mesmo em relação aos outros, existindo portanto a perspectiva de aumento de plantio dessa porta-enxerto em detrimento de outras, no Estado de São Paulo e, possivelmente, em outras regiões (Araújo, 1995).

\section{MATERIAL E MÉTODOS}

O presente trabalho foi instalado em área experimental do Departamento de Ciência do Solo, da Faculdade de Ciências Agronômicas - UNESP/Campus de Botucatu, Estado de São Paulo.

O experimento foi conduzido em condições de casa de vegetação sendo que os dados médios de temperatura e umidade foram registrados, durante a realização deste experimento, através de um termo-higrógrafo localizado a uma altura de 2,0 metros no centro da casa de vegetação. As temperaturas médias e umidade média para os meses de Março, Abril e Maio foram de $28,3,25,8$ e $23,4^{\circ} \mathrm{C}$ e $80,82,7$ e $91,5 \%$, respectivamente.

As sementes foram retiradas de frutos maduros colhidos no mês de junho de 1996 de plantas matrizes pertencentes ao Banco de Germoplasma do Centro de Citricultura Sylvio Moreira/IAC, localizado no município de Cordeirópolis-SP.
Cento e oitenta plantas de cada espécie, com aproximadamente 10 centímetros de altura, foram transferidas para recipientes de plástico com volume útil de 14 litros, permanecendo apoiadas nestes recipientes através de uma tampa de isopor com orifícios, onde o caule das plantas estavam envoltas por pedaços de espuma comum. Anterior ao processo de repicagem para as soluções tratamentos, as plantas passaram por aclimatação durante 20 dias, com o objetivo de minimizar os efeitos do estresse causado pelo processo de repicagem na absorção do alumínio. A solução nutritiva utilizada, baseada em recomendação de Furlani \& Furlani (1988), não continha alumínio e possuía a metade da concentração dos nutrientes. Após o período de 20 dias, trocou-se a solução nutritiva substituindo-a pela solução na concentração adequada ao experimento, descrita em Santos (1998), além da adição de cinco níveis de alumínio.

As concentrações de alumínio utilizadas foram: $0 ; 7,5 ; 15 ; 22,5$ e $30 \mathrm{mg} . \mathrm{L}^{-1}$ na forma de $\mathrm{AlCl}_{3} \cdot 6 \mathrm{H}_{2} \mathrm{O}$. O preparo da solução seguiu recomendação de Furlani \& Furlani (1988), tendo sua composição alterada, renovada quinzenalmente, oxigenada diariamente, sendo o $\mathrm{pH}$ da solução de cada tratamento corrigido também quinzenalmente para o valor $4 \pm 0,2$ com a adição de $\mathrm{HCl} 0,1 \mathrm{~N}$.

$\mathrm{Na}$ instalação do experimento para cada porta-enxerto adotou-se 0 delineamento estatístico inteiramente casualizado com 3 repetições, 5 concentrações de alumínio, em parcelas subdivididas. Cada parcela foi constituída de 12 plantas, e as plantas utilizadas em cada época de avaliação constituíram as subparcelas. Após o transplante para a solução nutritiva definitiva, as coletas das plantas destas subparcelas foram efetuadas a cada 14 dias, de acordo com Luchesi (1984): $1^{\text {a }}$ Coleta - 14 dias, $2^{\text {a }}$ Coleta - 28 dias, $3^{\text {a }}$ Coleta - 42 dias, $4^{\text {a }}$ Coleta - 56 dias, $5^{a}$ Coleta - 70 dias, $6^{a}$ Coleta - 84 dias após o transplante para a solução nutritiva. Realizaram-se análises de variância, sendo que as diferenças entre as médias dos tratamentos foram verificadas pelo teste de Tukey ao nível de $5 \%$ de probabilidade.

Após as coletas, as plantas foram destinadas à secagem em estufa com circulação forçada de ar com temperatura variando entre $65-70^{\circ} \mathrm{C}$ até peso constante, este material vegetal coletado foi pesado para obtenção dos pesos de matéria seca da parte aérea (folhas e caule) e raízes, depois moído e encaminhado ao 
Laboratório de Nutrição Mineral de Plantas do Departamento de Ciência do Solo da Faculdade de Ciências Agronômicas - UNESP/Campus de Botucatu, para a determinação analítica de $\mathrm{N}, \mathrm{P}$, $\mathrm{K}$, Ca, Mg, S, e Al segundo metodologia descrita por Malavolta et al. (1989).

\section{RESULTADOS E DISCUSSÃO}

Nas condições deste experimento, com relação ao parâmetro acúmulo de nutrientes, o limoeiro Cravo não foi influenciado pelos níveis de alumínio tanto quanto o citrumeleiro Swingle concordando em parte com Pompeu Júnior (1991), que menciona a superioridade de adaptação do primeiro porta-enxerto às diferentes condições de estresse. Portanto, os resultados obtidos nestas condições, mostram que o citrumeleiro Swingle mostrou-se sensível ao alumínio já no nível de $7,5 \mathrm{mg} \mathrm{L}^{-1}$ em solução nutritiva. É provável que a redução no acúmulo dos nutrientes nas plantas tenha ocorrido em conseqüência da própria redução no desenvolvimento das mesmas, devido às alterações fisiológicas provocadas pelo alumínio conforme relatos de Foy (1976) e Foy et al. (1978). Desta forma verificou-se que com adição de alumínio em solução nutritiva, que afetou o acúmulo de nutrientes e, aliado a sua ação de forma direta na fisiologia das plantas provavelmente proporcionou reduções significa-tivas nos pesos das matérias seca das folhas, caule e raízes das plantas de citrumelo Swingle, de acordo com os resultados médios apresenta-dos nas TABELAS 1, 2 e 3. Observa-se nestas TABELAS que o peso da matéria seca do limoeiro

TABELA 1 - Resultados médios de peso de matéria seca de folhas de limoeiro Cravo e citrumeleiro Swingle sob diferentes níveis de $\mathrm{Al}$, em hidroponia.

\begin{tabular}{ccc}
\hline Tratamentos & \multicolumn{2}{c}{ Porta-enxertos } \\
\cline { 2 - 3 } $\mathrm{mg} \mathrm{L}^{-1} \mathrm{Al}$ & limoeiro Cravo & $\mathrm{c}$. Swingle \\
0 & $1,38 \mathrm{~A}$ & $0,46 \mathrm{~A}$ \\
7,5 & $1,34 \mathrm{~A}$ & $0,35 \mathrm{~B}$ \\
15 & $1,39 \mathrm{~A}$ & $0,35 \mathrm{~B}$ \\
22,5 & $1,31 \mathrm{~A}$ & $0,30 \mathrm{~B}$ \\
30 & $1,10 \mathrm{~A}$ & $0,31 \mathrm{~B}$ \\
\hline Teste F & $\mathrm{NS}$ & $* \star$ \\
CV (\%) & 38,34 & 20,62 \\
\hline
\end{tabular}

Médias seguidas da mesma letra, nas colunas, não diferem entre si pelo Teste de Tukey ao nível de $5 \%$.
Cravo não foi influenciado significativamente pelos níveis de alumínio, não corroborando com os resultados obtidos por Nogueira et al. (1989), os quais concluíram que a presença do alumínio diminuiu os pesos das matérias seca das folhas e raízes do limoeiro Cravo, tangerineira Sunki e tangelo Orlando, enquanto Magalhães (1987) relata que o alumínio prejudicou severamente 0 desenvolvimento e crescimento do limoeiro Cravo, reduzindo o peso da matéria seca da parte aérea quando os níveis de alumínio variaram entre 20 e $80 \mathrm{mg} \mathrm{L}^{-1}$. Provavelmente, esta diferença significativa não observada deve-se ao elevado coeficiente de variação obtido nas análises estatísticas para este porta-enxerto, explicado talvez pela incidência de doença

TABELA 2 - Resultados médios de peso de matéria seca de caule de limoeiro Cravo e citrumeleiro Swingle sob diferentes níveis de $\mathrm{Al}$, em hidroponia.

\begin{tabular}{ccc}
\hline Tratamentos & \multicolumn{2}{c}{ Porta-enxertos } \\
\cline { 2 - 3 } mg L-1 $^{-1} \mathrm{Al}$ & limoeiro Cravo & c. Swingle \\
0 & $1,07 \mathrm{~A}$ & $0,57 \mathrm{~A}$ \\
7,5 & $1,05 \mathrm{~A}$ & $0,46 \mathrm{~B}$ \\
15 & $1,13 \mathrm{~A}$ & $0,41 \mathrm{~B}$ \\
22,5 & $1,02 \mathrm{~A}$ & $0,38 \mathrm{~B}$ \\
30 & $0,86 \mathrm{~A}$ & $0,37 \mathrm{~B}$ \\
\hline Teste F & $\mathrm{NS}$ & $* *$ \\
CV (\%) & 36,77 & 22 \\
\hline
\end{tabular}

Médias seguidas da mesma letra, nas colunas, não diferem entre si pelo Teste de Tukey ao nível de $5 \%$.

TABELA 3 - Resultados médios de peso de matéria seca de raízes de limoeiro Cravo e citrumeleiro Swingle sob diferentes níveis de Al, em hidroponia.

\begin{tabular}{ccc}
\hline Tratamentos & \multicolumn{2}{c}{ Porta-enxertos } \\
\cline { 2 - 3 } mg L-1 Al $^{-1}$ & limoeiro Cravo & c. Swingle \\
0 & $0,85 \mathrm{~A}$ & $0,52 \mathrm{~A}$ \\
7,5 & $0,90 \mathrm{~A}$ & $0,42 \mathrm{AB}$ \\
15 & $0,95 \mathrm{~A}$ & $0,37 \mathrm{~B}$ \\
22,5 & $0,81 \mathrm{~A}$ & $0,36 \mathrm{~B}$ \\
30 & $0,77 \mathrm{~A}$ & $0,34 \mathrm{~B}$ \\
\hline Teste F & $\mathrm{NS}$ & $\star \star$ \\
CV (\%) & 52,73 & 27,03 \\
\hline
\end{tabular}

Médias seguidas da mesma letra, nas colunas, não diferem entre si pelo Teste de Tukey ao nível de $5 \%$. 
fúngica no sistema radicular. Salienta-se porém que, apesar do rápido controle desta doença ocorreram reflexos negativos no crescimento do limoeiro Cravo, bem como no acúmulo de nutrientes relatados a seguir. Observou-se também, nestas condições, que os valores dos pesos de matéria seca tiveram seus valores aumentados durante o período de condução do experimento.

Os resultados médios do acúmulo de $\mathrm{N}$ na parte aérea e raízes das plantas de limão Cravo não foram influenciados significativamente pelos diferentes tratamentos com alumínio (TABELA 4). Apesar dos tratamentos não influenciarem neste acúmulo, observa-se tendência das plantas de limão Cravo dos tratamentos de 22,5 e $30 \mathrm{mg} \mathrm{L}^{-1}$ apresentarem menor acúmulo de $\mathrm{N}$ na parte aérea e raízes, verificando ainda, que na parte aérea o acúmulo de $\mathrm{N}$ foi maior que nas raízes. Este comportamento não foi observado por Nogueira et al. (1989), o qual relata que o cultivar Orlando acumulou mais $\mathrm{N}$ na raiz quando comparado à parte aérea. Considerando as coletas também como tratamento, observa-se, na TABELA 5 que houve acúmulo maior de $\mathrm{N}$ aos 84 dias, na parte aérea e raízes do porta-enxerto, mostrando a necessidade deste elemento no crescimento e desenvolvimento vegetal.

Não houve influência dos tratamentos de alumínio no acúmulo de $\mathrm{K}$ na parte aérea e raízes das plantas de limão Cravo, de acordo com os resultados médios mostrados na TABELA 6 . Também observa-se, para o mesmo portaenxerto, maior acúmulo de $\mathrm{K}$ no decorrer dos 84 dias de coletas (TABELA 7), embora havendo diminuição do acúmulo na parte aérea na última coleta. Nas raízes, o acúmulo de $\mathrm{K}$ foi menor que na parte aérea, mas também apresentou maior acúmulo nas últimas coletas (TABELA 7). Estes resultados concordam com os obtidos por Vasconcellos (1987) e Lin \& Myhre (1990; 1991). $\mathrm{O}$ acúmulo de $\mathrm{Mg}$ e $\mathrm{S}$ na parte aérea e raízes das plantas de limão Cravo não foi significativamente afetado pelos tratamentos com alumínio, conforme mostram os resultados médios nas TABELAS 8 e 10, respectivamente. Entretanto, pode-se observar para ambos maior acúmulo de $\mathrm{Mg}$ e $\mathrm{S}$ aos 84 dias tanto na parte aérea como nas raízes, sendo este maior na parte aérea (TABELAS 9 e 11). Os resultados obtidos para o limoeiro Cravo concordam com os obtidos por Lin \& Myhre (1990; 1991), que relatam uma alteração não significativa nos valores de acúmulo de $\mathrm{Mg}$ com a elevação nos níveis de alumínio em porta-enxertos cítricos. De acordo com Sanches \& Salinas (1981) o alumínio também interfere de forma não significativa no nível de $\mathrm{S}$ das plantas em condições de solos ácidos.

Com relação ao porta-enxerto citrumeleiro Swingle, a TABELA 4 mostra que o alumínio influenciou o acúmulo de $\mathrm{N}$ na parte aérea e nas raízes, observado a partir de $7,5 \mathrm{mg} \mathrm{L}^{-1}$, sendo apenas diferente significativamente do acúmulo apresentado pelas plantas do tratamento testemunha. $\mathrm{O}$ acúmulo de $\mathrm{N}$ foi maior na parte aérea aos 84 dias e, além disso observa-se também que houve aumento significativo deste acúmulo nas raízes, da primeira para a sexta coleta (TABELA 5). Este resultado é confirmado pelo trabalho de Vasconcellos (1987).

TABELA 4 - Resultados médios de acúmulo de $\mathrm{N}(\mathrm{mg})$ na parte aérea e raízes de limoeiro Cravo e citrumeleiro Swingle sob diferentes níveis de Al, em hidroponia.

\begin{tabular}{ccccc}
\hline Tratamento & limoeiro Cravo & c. Swingle & limoeiro Cravo & c. Swingle \\
\cline { 2 - 5 } mg L-1 Al & Parte Aérea & Parte Aérea & Raízes & Raízes \\
0 & $49,51 \mathrm{~A}$ & $23,96 \mathrm{~A}$ & $13,61 \mathrm{~A}$ & $10,79 \mathrm{~A}$ \\
7,5 & $42,28 \mathrm{~A}$ & $17,87 \mathrm{AB}$ & $14,64 \mathrm{~A}$ & $6,50 \mathrm{~B}$ \\
15 & $42,89 \mathrm{~A}$ & $16,44 \mathrm{~B}$ & $14,43 \mathrm{~A}$ & $5,94 \mathrm{~B}$ \\
22,5 & $35,38 \mathrm{~A}$ & $13,66 \mathrm{~B}$ & $12,08 \mathrm{~A}$ & $5,52 \mathrm{~B}$ \\
\hline 30 & $27,95 \mathrm{~A}$ & $13,31 \mathrm{~B}$ & $11,27 \mathrm{~A}$ & $5,13 \mathrm{~B}$ \\
\hline Teste F & $\mathrm{NS}$ & $* *$ & $\mathrm{NS}$ & $* *$ \\
CV (\%) & 52,49 & 36,98 & 55,54 & 28,36 \\
\hline
\end{tabular}

Médias seguidas da mesma letra, nas colunas, não diferem entre si pelo Teste de Tukey ao nível de 5\%. 
TABELA 5 - Resultados médios de acúmulo de $\mathrm{N}(\mathrm{mg})$ na parte aérea e raízes de limoeiro Cravo e citrumeleiro Swingle sob diferentes níveis de Al e coletas, em hidroponia.

\begin{tabular}{lcccc}
\hline \multirow{2}{*}{$\begin{array}{c}\text { Intervalo } \\
\text { entre Coletas }\end{array}$} & limoeiro Cravo & C. Swingle & limoeiro Cravo & C. Swingle \\
\cline { 2 - 5 } 14 dias & Parte Aérea & Parte Aérea & Raízes & Raízes \\
\hline 28 dias & $35,00 \mathrm{~A}$ & $14,43 \mathrm{~B}$ & $9,18 \mathrm{C}$ & $4,50 \mathrm{C}$ \\
$\mathbf{4 2}$ dias & $37,91 \mathrm{AB}$ & $16,65 \mathrm{AB}$ & $10,60 \mathrm{C}$ & $5,36 \mathrm{C}$ \\
$\mathbf{5 6}$ dias & $40,75 \mathrm{ABC}$ & $16,91 \mathrm{AB}$ & $12,29 \mathrm{BC}$ & $6,34 \mathrm{BC}$ \\
70 dias & $37,70 \mathrm{~B}$ & $15,43 \mathrm{~B}$ & $12,60 \mathrm{BC}$ & $6,33 \mathrm{~B}$ \\
84 dias & $41,91 \mathrm{BC}$ & $18,47 \mathrm{AB}$ & $14,16 \mathrm{~B}$ & $8,12 \mathrm{AB}$ \\
\hline Teste $\mathrm{F}$ & $44,95 \mathrm{C}$ & $20,40 \mathrm{~A}$ & $20,41 \mathrm{~A}$ & $10,00 \mathrm{~A}$ \\
CV $(\%)$ & $* *$ & $* *$ & $* *$ & $* *$ \\
\hline
\end{tabular}

Médias seguidas da mesma letra, nas colunas, não diferem entre si pelo Teste de Tukey ao nível de 5\%.

TABELA 6 - Resultados médios de acúmulo de $\mathrm{K}(\mathrm{mg})$ na parte aérea e raízes de limoeiro Cravo e citrumeleiro Swingle sob diferentes níveis de Al, em hidroponia.

\begin{tabular}{ccccc}
\hline Tratamento & limoeiro Cravo & c. Swingle & limoeiro Cravo & c. Swingle \\
\cline { 2 - 5 } mg L-1 Al & Parte Aérea & Parte Aérea & Raízes & Raízes \\
0 & $33,03 \mathrm{~A}$ & $9,96 \mathrm{~A}$ & $1,14 \mathrm{~A}$ & $0,74 \mathrm{~A}$ \\
7,5 & $22,55 \mathrm{~A}$ & $6,25 \mathrm{~B}$ & $0,95 \mathrm{~A}$ & $0,42 \mathrm{~B}$ \\
15 & $26,74 \mathrm{~A}$ & $6,17 \mathrm{~B}$ & $0,96 \mathrm{~A}$ & $0,38 \mathrm{~B}$ \\
22,5 & $26,23 \mathrm{~A}$ & $5,37 \mathrm{~B}$ & $0,75 \mathrm{~A}$ & $0,36 \mathrm{~B}$ \\
\hline 30 & $22,56 \mathrm{~A}$ & $5,76 \mathrm{~B}$ & $0,74 \mathrm{~A}$ & $0,36 \mathrm{~B}$ \\
\hline Teste F & $\mathrm{NS}$ & $* *$ & $\mathrm{NS}$ & $* *$ \\
CV (\%) & 49,70 & 25,86 & 108,01 & 44,08 \\
\hline
\end{tabular}

Médias seguidas da mesma letra, nas colunas, não diferem entre si pelo Teste de Tukey ao nível de $5 \%$.

Conforme mostram as TABELAS 6 e 8 a partir de $7,5 \mathrm{mg} \mathrm{L}^{-1}$ houve diminuição no acúmulo de $\mathrm{K}$ e $\mathrm{Mg}$ na parte aérea e raízes do citrumeleiro swingle, sendo estatisticamente diferente somente do acúmulo apresentado pelas plantas do tratamento testemunha. Embora haja este efeito de tratamento, verifica-se maior acúmulo de $\mathrm{K}$ e $\mathrm{Mg}$ na parte aérea comparado às raízes da primeira para a sexta coleta (TABELAS 7 e 9), respectivamente. Estes resultados concordam com os obtidos por Vasconcellos (1987), discordando entretanto, daqueles encontrados por Lin \& Myhre (1990; 1991), uma vez que estes obtiveram que os teores de $\mathrm{K}$ permaneceram inalterados, por Pavan \& Bingham (1982) em trabalhos de pesquisa com cafeeiros e Nogueira et al. (1989) com porta-enxertos cítricos, que observaram aumento na absorção de $\mathrm{K}$ quando se elevaram os níveis de alumínio. De acordo com Grassi Filho (1997), conforme há o avanço em idade do sistema radicular, diminui a velocidade média de absorção e aumenta o Km da enzima responsável por esta, porém a taxa de absorção se eleva com a idade devido ao efeito do número de raízes, diminuindo a absorção de $\mathrm{K}$ por metro linear de raiz. Assim sendo, nas condições deste experimento, o alumínio afetou o peso de matéria seca das raízes no caso do citrumeleiro Swingle, podendo portanto ter influenciado na absorção de $\mathrm{K}$. Além disso, o pH baixo também pode ter influenciado na absorção, pois favorece a competição com $\mathrm{H}$. 
TABELA 7 - Resultados médios de acúmulo de $\mathrm{K}(\mathrm{mg})$ na parte aérea e raízes de limoeiro Cravo e citrumeleiro Swingle sob diferentes níveis de Al e coletas, em hidroponia.

\begin{tabular}{lcccc}
\hline \multirow{2}{*}{ Intervalo } & limoeiro Cravo & c. Swingle & limoeiro Cravo & c. Swingle \\
\cline { 2 - 5 } entre Coletas & Parte Aérea & Parte Aérea & Raízes & Raízes \\
\hline 14 dias & $23,70 \mathrm{~B}$ & $6,53 \mathrm{~B}$ & $0,89 \mathrm{~B}$ & $0,24 \mathrm{C}$ \\
\hline 28 dias & $26,20 \mathrm{AB}$ & $7,90 \mathrm{~A}$ & $0,75 \mathrm{~B}$ & $0,34 \mathrm{C}$ \\
\hline 2 dias & $28,46 \mathrm{~A}$ & $6,90 \mathrm{AB}$ & $0,65 \mathrm{~B}$ & $0,37 \mathrm{BC}$ \\
\hline 56 dias & $26,35 \mathrm{AB}$ & $6,33 \mathrm{~B}$ & $0,53 \mathrm{~B}$ & $0,43 \mathrm{~B}$ \\
70 dias & $27,15 \mathrm{~A}$ & $6,29 \mathrm{~B}$ & $1,24 \mathrm{~B}$ & $0,59 \mathrm{AB}$ \\
84 dias & $25,47 \mathrm{AB}$ & $6,27 \mathrm{~B}$ & $1,39 \mathrm{~A}$ & $0,75 \mathrm{~A}$ \\
\hline Teste $\mathrm{F}$ & $* *$ & $* *$ & $* *$ & $* *$ \\
CV $(\%)$ & 12,13 & 17,76 & 41,76 & 44,41 \\
\hline
\end{tabular}

Médias seguidas da mesma letra, nas colunas, não diferem entre si pelo Teste de Tukey ao nível de 5\%.

TABELA 8 - Resultados médios de acúmulo de $M g(\mathrm{mg})$ na parte aérea e raízes de limoeiro Cravo e citrumeleiro Swingle sob diferentes níveis de Al, em hidroponia.

\begin{tabular}{ccccc}
\hline Tratamento & limoeiro Cravo & c. Swingle & limoeiro Cravo & c. Swingle \\
\cline { 2 - 5 } $\mathrm{mg} \mathrm{L}^{-1} \mathrm{Al}$ & Parte Aérea & Parte Aérea & Raízes & Raízes \\
0 & $3,02 \mathrm{~A}$ & $1,53 \mathrm{~A}$ & $0,22 \mathrm{~A}$ & $0,15 \mathrm{~A}$ \\
7,5 & $2,89 \mathrm{~A}$ & $1,18 \mathrm{~B}$ & $0,20 \mathrm{~A}$ & $0,09 \mathrm{~B}$ \\
15 & $2,99 \mathrm{~A}$ & $1,16 \mathrm{~B}$ & $0,21 \mathrm{~A}$ & $0,08 \mathrm{~B}$ \\
22,5 & $2,75 \mathrm{~A}$ & $1,01 \mathrm{~B}$ & $0,18 \mathrm{~A}$ & $0,09 \mathrm{~B}$ \\
\hline 30 & $2,54 \mathrm{~A}$ & $0,98 \mathrm{~B}$ & $0,21 \mathrm{~A}$ & $0,08 \mathrm{~B}$ \\
\hline Teste $\mathrm{F}$ & $\mathrm{NS}$ & $* *$ & $\mathrm{NS}$ & $* *$ \\
CV $(\%)$ & 28,34 & 23,94 & 55,29 & 42,67 \\
\hline
\end{tabular}

Médias seguidas da mesma letra, nas colunas, não diferem entre si pelo Teste de Tukey ao nível de 5\%.

Estes resultados encontrados para $\mathrm{OMg}$ corroboram com os encontrados por Haynes (1982) relatando que a toxicidade de alumínio é freqüentemente acompanhada por baixas concentrações de $\mathrm{Mg}$ nos tecidos de diversas espécies de plantas, como relatado por Pavan \& Bingham (1982) em cafeeiros, Vasconcellos (1987) e Nogueira et al. (1989) em porta-enxertos cítricos, e contrariando os encontrados por Lin \& Myhre (1990; 1991).

Os resultados médios de acúmulo de $\mathrm{S}$ apresentados na TABELA 10 mostram que os tratamentos de alumínio influenciaram significativamente no acúmulo de $S$ na parte aérea e raízes do citrumeleiro Swingle a partir de $7,5 \mathrm{mg} \mathrm{L}^{-1}$ de alumínio, não se verificando diferença significativa entre os demais tratamentos e, corroborando com os resultados encontrados por Vasconcellos (1987). Verifica-se tanto para a parte aérea quanto para as raízes do citrumeleiro Swingle e limoeiro Cravo que o acúmulo de $\mathrm{S}$ foi constante no decorrer de todas as coletas, tendo as raízes em contrapartida acúmulo significativamente elevado da primeira à ultima coleta, embora sendo menor que o da parte aérea (TABELA 11).

Segundo Foy (1976) o alumínio pode interferir na absorção, transporte e utilização de vários elementos como o $\mathrm{P}$, concordando com Pavan \& Bingham (1982) e Nogueira et al. (1989), bem como o cálcio conforme Vasconcellos (1987) e Nogueira et al. (1989). 
TABELA 9 - Resultados médios de acúmulo de $M g(\mathrm{mg})$ na parte aérea e raízes de limoeiro Cravo e citrumeleiro Swingle sob diferentes níveis de Al e coletas, em hidroponia.

\begin{tabular}{lcccc}
\hline \multirow{2}{*}{$\begin{array}{c}\text { Intervalo } \\
\text { entre Coletas }\end{array}$} & limoeiro Cravo & c. Swingle & limoeiro Cravo & C. Swingle \\
\cline { 2 - 5 } 14 dias & Parte Aérea & Parte Aérea & Raízes & Raízes \\
\hline 28 dias & $2,68 \mathrm{~B}$ & $0,95 \mathrm{~B}$ & $0,19 \mathrm{BC}$ & $0,07 \mathrm{~B}$ \\
42 dias & $2,79 \mathrm{AB}$ & $1,19 \mathrm{~A}$ & $0,16 \mathrm{C}$ & $0,07 \mathrm{~B}$ \\
56 dias & $3,00 \mathrm{~A}$ & $1,16 \mathrm{AB}$ & $0,27 \mathrm{~A}$ & $0,14 \mathrm{~A}$ \\
70 dias & $2,64 \mathrm{~B}$ & $1,16 \mathrm{AB}$ & $0,15 \mathrm{C}$ & $0,08 \mathrm{~B}$ \\
84 dias & $2,89 \mathrm{AB}$ & $1,28 \mathrm{~A}$ & $0,21 \mathrm{~B}$ & $0,10 \mathrm{AB}$ \\
\hline Teste $\mathrm{F}$ & $3,01 \mathrm{~A}$ & $1,29 \mathrm{~A}$ & $0,24 \mathrm{AB}$ & $0,14 \mathrm{~A}$ \\
CV $(\%)$ & $* *$ & $* *$ & $* *$ & $* *$ \\
\hline
\end{tabular}

Médias seguidas da mesma letra, nas colunas, não diferem entre si pelo Teste de Tukey ao nível de 5\%.

TABELA 10 - Resultados médios de acúmulo de $S(\mathrm{mg})$ na parte aérea e raízes de limoeiro Cravo e citrumeleiro Swingle sob diferentes níveis de Al, em hidroponia.

\begin{tabular}{ccccc}
\hline Tratamento & limoeiro Cravo & c. Swingle & limoeiro Cravo & c. Swingle \\
\cline { 2 - 5 } $\mathrm{mg} \mathrm{L}^{-1} \mathrm{Al}$ & Parte Aérea & Parte Aérea & Raízes & Raízes \\
0 & $2,41 \mathrm{~A}$ & $1,36 \mathrm{~A}$ & $0,65 \mathrm{~A}$ & $0,61 \mathrm{~A}$ \\
7,5 & $2,14 \mathrm{~A}$ & $0,83 \mathrm{~B}$ & $0,80 \mathrm{~A}$ & $0,50 \mathrm{AB}$ \\
15 & $2,35 \mathrm{~A}$ & $0,83 \mathrm{~B}$ & $0,71 \mathrm{~A}$ & $0,44 \mathrm{AB}$ \\
22,5 & $2,09 \mathrm{~A}$ & $0,65 \mathrm{~B}$ & $0,57 \mathrm{~A}$ & $0,42 \mathrm{AB}$ \\
30 & $1,72 \mathrm{~A}$ & $0,64 \mathrm{~B}$ & $0,61 \mathrm{~A}$ & $0,40 \mathrm{~B}$ \\
\hline Teste F & $\mathrm{NS}$ & $* *$ & $\mathrm{NS}$ & $* *$ \\
CV $(\%)$ & 45,05 & 39,66 & 52,10 & 36,89 \\
\hline
\end{tabular}

Médias seguidas da mesma letra, nas colunas, não diferem entre si pelo Teste de Tukey ao nível de 5\%.

As interações mais importantes, que se pode verificar são alumínio e fósforo e alumínio e cálcio sendo que, os demais elementos não mostram correlação com a absorção de alumínio, conforme também relataram Lin \& Myhre (1991). Portanto, de acordo com os resultados médios apresentados para o P na TABELA 12, observase que houve influência significativa dos tratamentos de alumínio no acúmulo de $\mathrm{P}$ na parte aérea e raízes das plantas de citrumelo Swingle. Constata-se também aumento significativo do fósforo nas raízes com o aumento dos níveis de alumínio da primeira para a última coleta, apesar dos valores de $P$ serem maiores na parte aérea (TABELA 13). Para o limoeiro Cravo verifica-se o mesmo comportamento, embora o alumínio não tenha influenciado significativamente no acúmulo deste (TABELAS 12 e 13). Para Lin \& Myhre (1991) não houve correlação aparente entre a concentração de fósforo nas raízes e na parte aérea com a tolerância dos porta-enxertos ao alumínio.

De acordo com Pavan \& Bingham (1982) em trabalho com cafeeiro, a percentagem de $P$ que permaneceu nas raízes aumentou e na parte aérea diminuiu com o aumento dos níveis de alumínio. McCormick \& Borden (1972), através de técnicas fotomicrográficas observaram sítios de fixação de fosfato pelo alumínio dentro das raízes das plantas, concluindo que a interação alumínio-fosfato pareceu estar associada com a parede celular e membrana citoplasmática da 
TABELA 11 - Resultados médios de acúmulo de $S(\mathrm{mg})$ na parte aérea e raízes de limoeiro Cravo e citrumeleiro Swingle sob diferentes níveis de Al e coletas, em hidroponia.

\begin{tabular}{lcccc}
\hline \multirow{2}{*}{$\begin{array}{c}\text { Intervalo } \\
\text { entre Coletas }\end{array}$} & limoeiro Cravo & c. Swingle & limoeiro Cravo & c. Swingle \\
\cline { 2 - 5 } 14 dias & Parte Aérea & Parte Aérea & Raízes & Raízes \\
\hline 28 dias & $1,94 \mathrm{~B}$ & $0,75 \mathrm{~A}$ & $0,57 \mathrm{C}$ & $0,27 \mathrm{D}$ \\
\hline 2 dias & $2,79 \mathrm{~A}$ & $0,83 \mathrm{~A}$ & $0,17 \mathrm{D}$ & $0,41 \mathrm{C}$ \\
56 dias & $2,20 \mathrm{~B}$ & $0,86 \mathrm{~A}$ & $0,75 \mathrm{~B}$ & $0,48 \mathrm{BC}$ \\
70 dias & $1,99 \mathrm{~B}$ & $0,85 \mathrm{~A}$ & $0,72 \mathrm{BC}$ & $0,47 \mathrm{~B}$ \\
\hline 4 dias & $1,93 \mathrm{~B}$ & $0,94 \mathrm{~A}$ & $0,85 \mathrm{AB}$ & $0,58 \mathrm{AB}$ \\
\hline Teste $\mathrm{F}$ & $1,99 \mathrm{~B}$ & $0,95 \mathrm{~A}$ & $0,95 \mathrm{~A}$ & $0,64 \mathrm{~A}$ \\
CV $(\%)$ & $* *$ & $\mathrm{NS}$ & $* *$ & $* *$ \\
\hline
\end{tabular}

Médias seguidas da mesma letra, nas colunas, não diferem entre si pelo Teste de Tukey ao nível de 5\%.

TABELA 12 - Resultados médios de acúmulo de $P(\mathrm{mg})$ na parte aérea e raízes de limoeiro Cravo e citrumeleiro Swingle sob diferentes níveis de Al, em hidroponia.

\begin{tabular}{ccccc}
\hline Tratamento & limoeiro Cravo & c. Swingle & limoeiro Cravo & c. Swingle \\
\cline { 2 - 5 } $\mathrm{mg} \mathrm{L}^{-1} \mathrm{Al}$ & Parte Aérea & Parte Aérea & Raízes & Raízes \\
0 & $1,20 \mathrm{~A}$ & $0,73 \mathrm{~A}$ & $0,62 \mathrm{~A}$ & $0,53 \mathrm{~A}$ \\
7,5 & $1,36 \mathrm{~A}$ & $0,52 \mathrm{~B}$ & $0,75 \mathrm{~A}$ & $0,41 \mathrm{~B}$ \\
15 & $1,33 \mathrm{~A}$ & $0,54 \mathrm{~B}$ & $0,77 \mathrm{~A}$ & $0,41 \mathrm{~B}$ \\
22,5 & $1,27 \mathrm{~A}$ & $0,44 \mathrm{~B}$ & $0,69 \mathrm{~A}$ & $0,43 \mathrm{~B}$ \\
30 & $1,06 \mathrm{~A}$ & $0,48 \mathrm{~B}$ & $0,68 \mathrm{~A}$ & $0,42 \mathrm{~B}$ \\
\hline Teste F & $\mathrm{NS}$ & $* *$ & $\mathrm{NS}$ & $* *$ \\
CV $(\%)$ & 38,33 & 26,03 & 45,83 & 20,04 \\
\hline
\end{tabular}

Médias seguidas da mesma letra, nas colunas, não diferem entre si pelo Teste de Tukey ao nível de $5 \%$.

epiderme e células do córtex. Vasconcellos (1987) e Grassi Filho (1997) relatam que uma redução na extração de $P$ pelos porta-enxertos cítricos pode também ser observada, provavelmente devido à menor disponibilidade desse elemento em $\mathrm{pH}$ ácido. Esta redução na extração também pode estar relacionada com a complexação do $\mathrm{P}$ pelo alumínio no substrato externo à raiz diminuindo a absorção do mesmo (McCormick \& Borden, 1972).

De acordo com os resultados médios mostrados na TABELA 14, os níveis de alumínio influenciaram significativamente no acúmulo de Ca na parte aérea e raízes do citrumeleiro Swingle e parte aérea do limoeiro Cravo, havendo aumento no acúmulo de $\mathrm{Ca}$ na parte aérea e raízes deste e na parte aérea do limoeiro Cravo, de acordo com o aumento do acúmulo de Al com o decorrer das coletas, sendo maior na parte aérea, sugerindo que as folhas do porta-enxerto foram o principal órgão armazenador de $\mathrm{Ca}$ (TABELA 15). Segundo Wagatsuma (1983) a absorção de alumínio pelas raízes, no caso do milho, diminuiu com o aumento da concentração de cálcio no meio de crescimento, parecendo portanto, que o alumínio compete com cálcio pelo mesmo sítio de absorção nas raízes. A afinidade do alumínio para os sítios de absorção pode ser considerado mais forte que o cálcio e, portanto para evitar a toxicidade de alumínio uma considerável concentração de cálcio comparada ao alumínio pode ser necessária. Para Haynes (1982), a toxicidade de alumínio é freqüentemente 
TABELA 13 - Resultados médios de acúmulo de $\mathrm{P}(\mathrm{mg})$ na parte aérea e raízes de limoeiro Cravo e citrumeleiro Swingle sob diferentes níveis de Al e coletas, em hidroponia.

\begin{tabular}{lcccc}
\hline \multirow{2}{*}{$\begin{array}{c}\text { Intervalo } \\
\text { entre Coletas }\end{array}$} & limoeiro Cravo & c. Swingle & limoeiro Cravo & c. Swingle \\
\cline { 2 - 5 } 14 dias & Parte Aérea & Parte Aérea & Raízes & Raízes \\
\hline 28 dias & $1,42 \mathrm{~A}$ & $0,63 \mathrm{~A}$ & $0,59 \mathrm{~B}$ & $0,33 \mathrm{D}$ \\
\hline 2 dias & $1,16 \mathrm{BC}$ & $0,65 \mathrm{~A}$ & $0,65 \mathrm{~B}$ & $0,44 \mathrm{~B}$ \\
56 dias & $1,19 \mathrm{ABC}$ & $0,54 \mathrm{AB}$ & $0,57 \mathrm{~B}$ & $0,39 \mathrm{C}$ \\
70 dias & $0,98 \mathrm{C}$ & $0,45 \mathrm{~B}$ & $0,61 \mathrm{~B}$ & $0,44 \mathrm{~B}$ \\
\hline 4 dias & $1,38 \mathrm{AB}$ & $0,49 \mathrm{~B}$ & $0,87 \mathrm{~A}$ & $0,51 \mathrm{AB}$ \\
\hline Teste $\mathrm{F}$ & $1,31 \mathrm{~B}$ & $0,49 \mathrm{~B}$ & $0,92 \mathrm{~A}$ & $0,53 \mathrm{~A}$ \\
$\mathrm{CV}(\%)$ & $* *$ & $\mathrm{NS}$ & $* *$ & $* *$ \\
\hline
\end{tabular}

Médias seguidas da mesma letra, nas colunas, não diferem entre si pelo Teste de Tukey ao nível de 5\%.

TABELA 14 - Resultados médios de acúmulo de $\mathrm{Ca}(\mathrm{mg})$ na parte aérea e raízes de limoeiro Cravo e citrumeleiro Swingle sob diferentes níveis de Al, em hidroponia.

\begin{tabular}{ccccc}
\hline Tratamento & limoeiro Cravo & c. Swingle & limoeiro Cravo & c. Swingle \\
\cline { 2 - 5 } $\mathrm{mg} \mathrm{L}^{-1} \mathrm{Al}$ & Parte Aérea & Parte Aérea & Raízes & Raízes \\
0 & $18,46 \mathrm{~A}$ & $9,84 \mathrm{~A}$ & $5,64 \mathrm{~A}$ & $2,12 \mathrm{~A}$ \\
7,5 & $13,14 \mathrm{AB}$ & $5,73 \mathrm{~B}$ & $3,53 \mathrm{~A}$ & $1,22 \mathrm{~B}$ \\
15 & $13,82 \mathrm{AB}$ & $5,59 \mathrm{~B}$ & $3,67 \mathrm{~A}$ & $1,03 \mathrm{~B}$ \\
22,5 & $12,15 \mathrm{AB}$ & $4,57 \mathrm{~B}$ & $2,84 \mathrm{~A}$ & $0,97 \mathrm{~B}$ \\
\hline 30 & $10,59 \mathrm{~B}$ & $4,78 \mathrm{~B}$ & $2,95 \mathrm{~A}$ & $0,94 \mathrm{~B}$ \\
\hline Teste F & $\mathrm{NS}$ & $* *$ & $\mathrm{NS}$ & $* *$ \\
CV $(\%)$ & 49,99 & 35,68 & 101,33 & 33,86 \\
\hline
\end{tabular}

Médias seguidas da mesma letra, nas colunas, não diferem entre si pelo Teste de Tukey ao nível de $5 \%$.

acompanhada por baixas concentrações de Ca no tecido das planta e, Grassi Filho (1997) cita que a competição de $\mathrm{Ca}$ com $\mathrm{H}$ em condições de $\mathrm{pH}$ ácido pode favorecer a absorção do segundo em detrimento do primeiro.

Entretanto, nas condições deste experimento, a concentração de cálcio utilizada foi suficiente para que $o$ alumínio não impedisse a sua absorção e transporte em maior quantidade para a parte aérea, em ambos os porta-enxertos. De acordo com Lin \& Myhre (1991), a concentração de cálcio em plantas cítricas pode não ser o principal fator relatado para os efeitos tóxicos do alumínio, salientando que os mecanismos de influência da concentração de alumínio no transporte de nutrientes das raízes para a parte aérea das plantas cítricas necessitam de mais investigação.

Para o citrumeleiro Swingle e limoeiro Cravo, os resultados médios apresentados na TABELA 16, mostram que os tratamentos de alumínio não influenciaram no acúmulo deste na parte aérea. Entretanto, nas raízes os resultados médios mostram que houve influência dos níveis de alumínio no seu acúmulo, sendo que as plantas do nível de $22,5 \mathrm{mg} \mathrm{L}^{-1}$ e $30 \mathrm{mg} \mathrm{L}^{-1}$ apresentaram o maior acúmulo, apesar de serem diferentes significativamente apenas do tratamento testemunha. Salienta-se também que, o acúmulo de alumínio foi maior nas raízes que na parte aérea do citrumeleiro Swingle e limoeiro Cravo e, os tratamentos de alumínio mostram influência apenas no acúmulo de $\mathrm{Al}$ nas raízes. 
TABELA 15 - Resultados médios de acúmulo de $\mathrm{Ca}(\mathrm{mg})$ na parte aérea e raízes de limoeiro Cravo e citrumeleiro Swingle sob diferentes níveis de Al e coletas, em hidroponia.

\begin{tabular}{lcccc}
\hline \multirow{2}{*}{$\begin{array}{c}\text { Intervalo } \\
\text { entre Coletas }\end{array}$} & limoeiro Cravo & c. Swingle & limoeiro Cravo & c. Swingle \\
\cline { 2 - 5 } 14 dias & Parte Aérea & Parte Aérea & Raízes & Raízes \\
\hline 28 dias & $12,18 \mathrm{C}$ & $4,21 \mathrm{C}$ & $2,83 \mathrm{C}$ & $0,77 \mathrm{C}$ \\
\hline 2 dias & $13,14 \mathrm{ABC}$ & $5,72 \mathrm{~B}$ & $3,44 \mathrm{~B}$ & $1,21 \mathrm{~B}$ \\
$\mathbf{5 6}$ dias & $14,46 \mathrm{AB}$ & $6,13 \mathrm{AB}$ & $3,85 \mathrm{AB}$ & $1,57 \mathrm{AB}$ \\
\hline 70 dias & $12,88 \mathrm{~B}$ & $6,42 \mathrm{AB}$ & $3,60 \mathrm{ABC}$ & $1,46 \mathrm{AB}$ \\
\hline 4 dias & $14,34 \mathrm{AB}$ & $6,97 \mathrm{AB}$ & $4,19 \mathrm{AB}$ & $1,80 \mathrm{~A}$ \\
\hline Teste $\mathrm{F}$ & $14,77 \mathrm{~A}$ & $7,16 \mathrm{~A}$ & $4,46 \mathrm{~A}$ & $0,73 \mathrm{C}$ \\
CV $(\%)$ & $* *$ & $* *$ & $* *$ & $* *$ \\
\hline
\end{tabular}

Médias seguidas da mesma letra, nas colunas, não diferem entre si pelo Teste de Tukey ao nível de $5 \%$.

TABELA 16 - Resultados médios de acúmulo de Al $(\mu \mathrm{g})$ na parte aérea e raízes de limoeiro Cravo e citrumeleiro Swingle sob diferentes níveis de Al, em hidroponia.

\begin{tabular}{ccccc}
\hline Tratamento & limoeiro Cravo & c. Swingle & limoeiro Cravo & c. Swingle \\
\cline { 2 - 5 } mg L-1 Al $^{-1}$ & Parte Aérea & Parte Aérea & Raízes & Raízes \\
\hline 0 & $365,23 \mathrm{~A}$ & $155,38 \mathrm{~A}$ & $403,26 \mathrm{~B}$ & $310,79 \mathrm{~B}$ \\
7,5 & $311,52 \mathrm{~A}$ & $124,80 \mathrm{~A}$ & $938,23 \mathrm{~A}$ & $743,28 \mathrm{~A}$ \\
15 & $379,48 \mathrm{~A}$ & $132,01 \mathrm{~A}$ & $1198,22 \mathrm{~A}$ & $767,27 \mathrm{~A}$ \\
22,5 & $348,12 \mathrm{~A}$ & $133,84 \mathrm{~A}$ & $1153,26 \mathrm{~A}$ & $898,82 \mathrm{~A}$ \\
30 & $297,24 \mathrm{~A}$ & $138,54 \mathrm{~A}$ & $1385,88 \mathrm{~A}$ & $832,65 \mathrm{~A}$ \\
\hline Teste F & $\mathrm{NS}$ & $\star *$ & $\mathrm{NS}$ & $* *$ \\
CV $(\%)$ & 45,53 & 38,48 & 47,89 & 34,59 \\
\hline
\end{tabular}

Médias seguidas da mesma letra, nas colunas, não diferem entre si pelo Teste de Tukey ao nível de 5\%.

TABELA 17 - Resultados médios de acúmulo de Al $(\mu \mathrm{g})$ na parte aérea e raízes de limoeiro Cravo e citrumeleiro Swingle sob diferentes níveis de Al e coletas, em hidroponia.

\begin{tabular}{lcccc}
\hline \multirow{2}{*}{$\begin{array}{c}\text { Intervalo } \\
\text { entre Coletas }\end{array}$} & limoeiro Cravo & c. Swingle & limoeiro Cravo & c. Swingle \\
\cline { 2 - 5 } 14 dias & Parte Aérea & Parte Aérea & Raízes & Raízes \\
\hline 28 dias & $177,67 \mathrm{C}$ & $67,44 \mathrm{C}$ & $695,79 \mathrm{C}$ & $397,63 \mathrm{C}$ \\
\hline 2 dias & $201,81 \mathrm{C}$ & $113,88 \mathrm{~B}$ & $936,57 \mathrm{BC}$ & $656,09 \mathrm{~B}$ \\
$\mathbf{5 6}$ dias & $365,64 \mathrm{~B}$ & $114,35 \mathrm{~B}$ & $931,03 \mathrm{BC}$ & $657,99 \mathrm{~B}$ \\
70 dias & $356,61 \mathrm{~B}$ & $140,59 \mathrm{~B}$ & $955,51 \mathrm{~B}$ & $628,53 \mathrm{~B}$ \\
\hline 4 dias & $467,47 \mathrm{~A}$ & $184,61 \mathrm{~A}$ & $1210,19 \mathrm{AB}$ & $904,09 \mathrm{~A}$ \\
\hline Teste $\mathrm{F}$ & $472,71 \mathrm{~A}$ & $200,63 \mathrm{~A}$ & $1365,54 \mathrm{~A}$ & $1019,04 \mathrm{~A}$ \\
CV $(\%)$ & $* *$ & $* *$ & $* *$ & $* *$ \\
\hline
\end{tabular}

Médias seguidas da mesma letra, nas colunas, não diferem entre si pelo Teste de Tukey ao nível de 5\%. 
Os resultados médios em função das coletas para os dois porta-enxertos, expressos na TABELA 17, mostram que no decorrer do crescimento e desenvolvimento das plantas houve acúmulo gradativo de alumínio na parte aérea e nas raízes, o qual foi aumentando até atingir um máximo, na última coleta.

\section{CONCLUSÕES}

A análise estatística dos dados, nas condições do presente estudo permitiram concluir que, com a adição a partir de $7,5 \mathrm{mg} \mathrm{L}^{-1}$ de alumínio em solução nutritiva, ocorreu redução significativa no acúmulo de macronutrientes associado a alterações fisiológicas e reduções significativas nos pesos da matéria seca das folhas, caule e raízes das plantas de citrumelo Swingle.

Deve-se destacar que, em virtude do lento crescimento apresentado pelos portaenxertos, há a necessidade de prolongar o intervalo entre avaliações adotado nestas condições, para que haja a possibilidade de se obter resultados mais conclusivos quanto ao efeito do alumínio na fisiologia destes.

\section{REFERÊNCIAS BIBLIOGRÁFICAS}

AMARO, A. A.; MAIA, M.L. Produção e comércio de laranja e suco no Brasil. Laranja, v.18, n.1, p.1-16, 1997.

ARAÚJO, J.R.G. Desenvolvimento e concentração mineral em três variedades-copa de citrus (Citrus spp), sob influência de diferentes porta-enxertos. Botucatu, 1995. 138 p. Dissertação (Mestrado) Faculdade de Ciências Agronômicas, Universidade Estadual Paulista.

FOY, C.D. Differential aluminum and manganese tolerances of plant species and varieties in acid soils. Ciência e Cultura, v.28, n.2, p.150-155, 1976.

FOY, C.D.; CHANEY, R.L.; WHITE, M.C. The physiology of metal toxicity in plants. Annual Review of Plant Physiology, v.29, p.511-566, 1978.

FURLANI, A.M.C.; FURLANI, P.R. Composição e $\mathrm{pH}$ de soluções nutritivas para estudos fisiológicos e seleção de plantas em condições adversas. Campinas: IAC, 1988. p.21-26. (Boletim Técnico, 121).

GRASSI FILHO, H. Nutrição mineral de plantas. Botucatu: UNESP, Faculdade de Ciências Agronômicas, Departamento de Ciência do Solo, 1997. 133p. (Apostila).

HAYNES, R.J. Effects of liming on phosphate availability in acid soils. Plant Soil, v.68, p.289-308, 1982.
LIN, Z.; MYHRE, D.L. Citrus root growth as affected by soil aluminum level under field conditions. Soil Science Society of America Journal, v.54, p.1340-1344, 1990.

LIN, Z.; MYHRE, D.L. Differential response of citrus rootstocks to aluminum levels in nutrient solutions: II. Plant mineral concentrations. Journal Plant Nutrition, v.14, p.1239-1254, 1991.

LUCHESI, A.A. Utilização prática da análise de crescimento vegetal. Anais da ESALQ, v.41, p.181-202, 1984.

MALAVOLTA, E.; VITTI, G.C.; OLIVEIRA, S.A. Avaliação do estado nutricional de plantas: princípios e aplicações. Piracicaba: Potafos, 1989. 208p.

MAGALHÃES, A.F.J. Tolerância de porta-enxertos de citrus ao alumínio. Revista Brasileira de Fruticultura, v.9, n.3, p.51-55, 1987.

McCORMICK, L.H.; BORDEN, F.Y. Phosphate fixation by aluminum in plant roots. Proceedings Soil Science Society American, v.36, n.5, p.709-802, 1972.

NOGUEIRA, S.S.; NAGAI, V.; CARELLI, M.L.C.; FAHL, J.J. Comportamento de porta-enxertos de citros em presença de alumínio. Pesquisa Agropecuária Brasileira, v.24, n.6, p.711-716, 1989.

PAVAN, M.A.; BINGHAM, F.T. Toxidez de alumínio em cafeeiros cultivados em solução nutritiva. Pesquisa Agropecuária Brasileira, v.17, n.9, p.1293-1302, 1982.

POMPEU JÚNIOR, J. Porta-enxertos. In: RODRIGUEZ, O. (Coord.) Citricultura brasileira. 2.ed. Campinas: Fundação Cargill, 1991. v.1, p.265-280.

SANCHES, P.A.; SALINAS, G. Low input technology for managing oxisols and ultisols in tropical America. Advances in Agronomy, v.34, p.280-406, 1981.

SANTOS, C.H. Influência de diferentes níveis de alumínio no desenvolvimento de dois portaenxertos cítricos em cultivo hidropônico. Botucatu, 1998. 134p. Dissertação (Mestrado) - Faculdade de Ciências Agronômicas, Universidade Estadual Paulista.

VASCONCELLOS, L.A.B.C. Comportamento de porta-enxertos de citros (Citrus spp) em três tipos de solos com diferentes níveis de alumínio e manganês. Piracicaba, 1987. 108p. Tese (Doutorado) - Escola Superior de Agricultura Luiz de Queiroz, Universidade de São Paulo.

WAGATSUMA, T. Characterization of absorption sites for aluminium in the roots. Soil Science Plant Nutrition, v.29, p.499-515, 1983.

Recebido para publicação em 11.11.98

Aceito para publicação em 06.08.99 